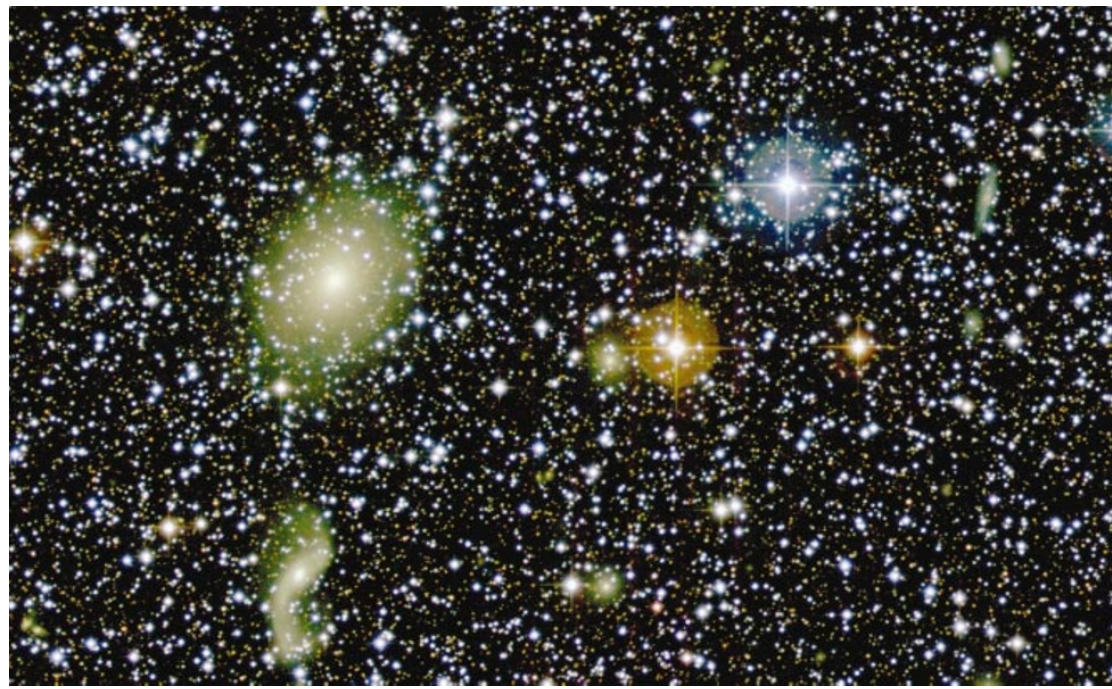

Dark secret: galaxy clusters such as ACO 3627 could help to unlock the mysteries of dark energy.

\title{
Cosmologists cluster to plot course towards dark energy
}

Geoff Brumfiel, Chicago

For five years, cosmologists and theoretical physicists have been wrestling with the bombshell discovery that a mysterious entity called 'dark energy' may be responsible for two-thirds of the energy and matter in the Universe.

Researchers are today no closer to defining dark energy, although they rely on it to explain the 1998 discovery that the Universe's expansion is accelerating, rather than slowing down under the influence of gravity, as had been widely assumed.

But they are at least closer to forging a plan to track it down. Last week, cosmologists met at the University of Chicago to hammer out a strategy to perform surveys of distant space that should glean more information about dark energy.

The plan involves constructing as many as 15 telescopes, including one in Antarctica, to work with existing ones in a bid to track thousands of clusters of galaxies. By looking at the distances between them, cosmologists hope to learn more about dark energy, which seems to make itself felt only between massive objects on a huge, intergalactic scale.

The survey will start by observing thousands of galaxy clusters up to 8 billion light years way. These clusters are observed now as they really were billions of years ago. If researchers compare the distances between these ancient clusters with those between clusters near our own galaxy — which can be observed pretty well as they are now - they may be able to establish how the clusters have been pushed apart, over time, by dark energy.

Finding and studying so many galactic clusters will be no small task, says John Carlstrom, a cosmologist at the University of Chicago who helped to organize the conference. Researchers will need to pick out clusters from the jumble of stars and galaxies in the night sky, and make precise measurements of the microwave radiation emitted by each cluster. Microwave radiation from space can easily be distorted by atmospheric water vapour, so much of the observation will be done by telescopes in very dry locations such as Antarctica.

Previous surveys of single galaxy clusters have taken several months using existing instruments, says Carlstrom. "My group has measured about 60 clusters in a decade," he says. But with more powerful telescopes and faster data processing, the time needed should fall sharply. A 3.5-metre-diameter microwave telescope due to start observations later this year in Owen's Valley, California, will do in months what used to take years, he says.

In the long term, Carlstrom's group is also planning an \$18-million, 8-metre-diameter microwave telescope at the South Pole, which will be able to do the same survey in a few hours when it starts operations in about four years' time. Other groups are planning similar projects in the Atacama Desert in Chile and on the mountaintops of Hawaii.

But questions remain about the effectiveness of the approach. For one thing, clusters of galaxies are unwieldy and awkward to define. 'A cluster isn't like a star — it doesn't have a well-defined edge," says August Evrard, a theoretical cosmologist at the University of Michigan in Ann Arbor.

Even so, Saul Perlmutter, an astronomer at the Lawrence Berkeley National Laboratory in California who led one of the groups that discovered dark energy, says the survey will represent an important step forward.
DNA lab welcomes Dalai Lama to Tibetan science community

\section{Erika Check}

Nyime Norbu has not seen his homeland, Tibet, since he and his family fled from there 30 years ago. So he was greatly moved last week when he came face-toface with the exiled spiritual leader of Tibet, the Dalai Lama, thousands of miles from their mutual home. What did they discuss? DNA sequencing, of course.

Norbu runs DNA-sequencing machines at the Whitehead Institute/MIT Center for Genome Research, and is one of about 20 Tibetans who work there. The centre has one of the largest communities of Tibetan workers in the Boston area, which is part of the reason for the Dalai Lama's visit on 13 September during a trip to Boston.

The centre's director, Eric Lander, hired the first of the Tibetans in 1991, when the centre was preparing to sequence the mouse genome. "We needed somebody who had some scientific background, who was also very careful," he says. He ended up hiring a Tibetan refugee who was both an artist and a former inspector at a milk factory.

He in turn recruited more Tibetans throughout the 1990s, making them the most strongly represented ethnic group in the Human Genome Project: although Tibetans make up only $0.1 \%$ of the world's population, it has been estimated that they represent about $10 \%$ of the project's workforce.

"Many of our Tibetan colleagues are refugees who have learned enough science and molecular biology to become extraordinarily valuable members of our team," says Lander.

Most of the centre's Tibetans escaped the country in the face of repression by China. "To have the Dalai Lama in front of me was a blessing - we were thrilled," says Norbu. "I have no words to explain how I felt.”

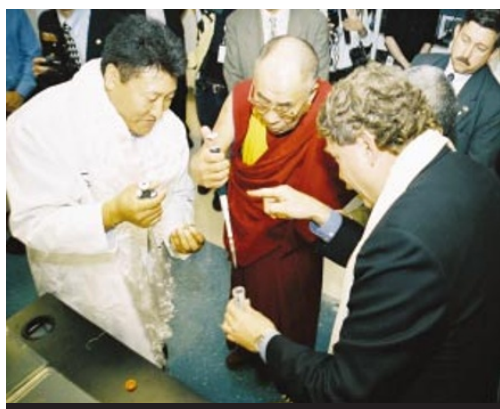

The Dalai Lama helps Eric Lander (right) and Yama Chopel (left) prepare mouse DNA. 